\title{
Management of L-dopa overdose in the competitive inhibition state
}

Marty Hinz'

Alvin Stein ${ }^{2}$

Ted Cole ${ }^{3}$

'Clinical Research, NeuroResearch Clinics, Inc., Cape Coral, FL, USA;

${ }^{2}$ Stein Orthopedic Associates, Plantation, FL, USA; ${ }^{3}$ Cole Center for Healing, Cincinnati, OH, USA
Correspondence: Marty Hinz NeuroResearch Clinics, Inc., 1008

Dolphin Dr, Cape Coral, FL 33904, USA

Tel +I 2186262220

Fax + I 2186261638

Email marty@hinzmd.com
This article was published in the following Dove Press journal:

Drug, Healthcare and Patient Safety

22 July 2014

Number of times this article has been viewed

Abstract: The amino acid L-3,4-dihydroxyphenylalanine (L-dopa) is prescribed for conditions where increased central and/or peripheral dopamine synthesis is desired. Its administration can establish dopamine concentrations higher than can be achieved from an optimal diet. Specific indications include Parkinson's disease and restless leg syndrome. The interaction between serotonin and dopamine exists in one of two distinctly different physiologic states: the endogenous state or the competitive inhibition state. Management with L-dopa in the competitive inhibition state is the focus of this paper. In the past, control of the competitive inhibition state was thought to be so difficult and complex that it was described in the literature as functionally "meaningless". When administering L-dopa without simultaneous administration of serotonin precursors, the patient is in the endogenous state. Experience gained with patient outcomes during endogenous L-dopa administration does not allow predictability of L-dopa outcomes in the competitive inhibition state. The endogenous approach typically increases the daily L-dopa dosing value in a linear fashion until symptoms of Parkinson's disease are under control. It is the novel observations made during treatment with the competitive inhibition state approach that L-dopa dosing values above or below the optimal therapeutic range are generally associated with the presence of the exact same Parkinson's disease symptoms with identical intensity. This recognition requires a novel approach to optimization of daily L-dopa dosing values from that used in the endogenous state. This paper outlines that novel approach through utilization of a pill stop. This approach enhances patient safety through its ability to prevent L-dopa overdose, while assisting in the establishment of the optimal therapeutic L-dopa daily dosing value.

Keywords: L-3,4-dihydroxyphenylalanine, L-dopa, levodopa, Parkinson's disease

\section{Introduction}

5-hydroxytryptophan (5-HTP) is a metabolite of L-tryptophan and the immediate precursor of serotonin. L-3,4-dihydroxyphenylalanine (L-dopa) is a metabolite of L-tyrosine and the immediate precursor of dopamine. Dopamine does not cross the blood-brain barrier. ${ }^{1} \mathrm{~L}$-dopa freely crosses the blood-brain barrier, then is synthesized into dopamine without biochemical feedback inhibition. ${ }^{2}$ Greater amounts of L-dopa need to be administered if increased synthesis of dopamine in the central nervous system is required..$^{3-12}$ L-tyrosine does not have this ability, due to norepinephrine biochemical feedback inhibition of tyrosine hydroxylase.

To understand the discussions contained herein, the concepts of the endogenous state and competitive inhibition state need to be defined. ${ }^{1,12-20}$

Humans taking no supplemental serotonin or dopamine amino acid precursors are in the endogenous state. The endogenous state also exists when L-dopa or 5-HTP 
is administered without adequate amounts of serotonin or dopamine precursors, respectively. The amino acid intermediates 5-HTP and L-dopa do not occur in the normal diet in amounts sufficient to produce a significant metabolic effect. The competitive inhibition state does not occur with normal or optimal food intake due to biochemical feedback inhibition of L-tyrosine and L-tryptophan. Their respective conversion to L-dopa and 5-HTP in a normal or optimal diet are inadequate to establish competitive inhibition. This limits the amount of dopamine and serotonin synthesized to levels less than are required to place the system into the competitive inhibition state. ${ }^{12-20}$ When daily dopamine and dopamine amino acid requirements are higher than can be achieved in a normal or optimal diet, the state is known as a relative nutritional deficiency. ${ }^{12}$

The concept of competitive inhibition between serotonin and dopamine is well known to science. Competitive inhibition is the interaction of serotonin and dopamine that may occur in synthesis, transport, and metabolism only when adequate and properly balanced amounts of serotonin and dopamine amino acid precursors are administered simultaneously. Full optimization of the competitive inhibition state requires simultaneous administration of properly balanced 5-HTP, L-dopa, L-tyrosine, a thiol (L-cysteine, glutathione, S-adenosylmethionine, or L-methionine), and cofactors (vitamin $\mathrm{C}$, pyridoxal phosphate, or calcium carbonate). To date, the only published methodology for optimization of the competitive inhibition state is Organic Cation Transporter Type 2 (OCT2) functional status determination. ${ }^{12-20}$

The focus of this paper is not L-dopa efficacy, which has been firmly established by numerous past studies; this paper focuses on management of L-dopa dosing utilizing a novel technique that identifies overdose in the competitive inhibition state relative to optimal daily dosing, and assists in identifying the optimal dosing range.

Administration of L-dopa in Parkinson's disease has been studied since the early $1960 \mathrm{~s} .{ }^{21}$ Since then, numerous side effects and adverse reactions have been documented., ${ }^{2,12}$ Most agree with the Mayo Clinic's observations that L-dopa is the most effective Parkinson's disease treatment available. ${ }^{22}$ Typically, other less effective drugs are used to control symptoms as long as possible prior to prescribing L-dopa. This delays the inevitable onset of progressive side effects and adverse reactions associated with concomitant administration of L-dopa and carbidopa (or benserazide). ${ }^{21}$

Past research documented the use of general decarboxylase inhibitors such as carbidopa and benserazide for the management of L-dopa-induced nausea. ${ }^{23,24}$ These drugs have no direct benefit in the management of Parkinson's disease symptoms. The primary reason for administering carbidopa or benserazide is to decrease daily L-dopa dosing requirement, thereby decreasing L-dopa-induced nausea. During L-dopa monotherapy (administration without a decarboxylase inhibitor), these side effects may prevent the patient from ingesting enough L-dopa to control symptoms. ${ }^{2}$

The enzyme L-aromatic amino acid decarboxylase (AAAD) catalyzes synthesis of serotonin and dopamine from 5-HTP and L-dopa, respectively. Through competitive inhibition of AAAD, carbidopa or benserazide compromises peripheral synthesis of serotonin and dopamine. This druginduced inhibition of peripheral AAAD-L-dopa metabolism leaves more L-dopa unmetabolized and available to freely cross the blood-brain barrier into the central nervous system. As a result, when carbidopa or benserazide is administered, lower L-dopa daily intake values are required to achieve the same central nervous system results. ${ }^{2}$

Carbidopa and benserazide also inhibit peripheral metabolism of 5-HTP to serotonin and can cause a drug-induced depletion of peripheral serotonin. Dopamine is metabolized to norepinephrine, which, in turn, is metabolized to epinephrine. The inhibition of dopamine synthesis may also deplete norepinephrine and epinephrine. Physicians may fail to recognize the signs, symptoms, adverse reactions, and side effects that result from this drug-induced peripheral depletion of serotonin, dopamine, norepinephrine, and/or epinephrine by carbidopa. It is known that inhibition of AAAD with drugs may induce life-threatening side effects, including myocardial infarction, neuroleptic malignant syndrome, agranulocytosis, hemolytic and nonhemolytic anemia, gastrointestinal bleeding, thrombocytopenia, and hypokalemia (Table 1). ${ }^{2,12}$

Hinz et $\mathrm{al}^{2,12}$ previously published papers demonstrating that L-dopa-induced nausea can be nutritionally managed by addressing serotonin and dopamine imbalance. Proper administration of 5-HTP with L-dopa effectively controls nausea, eliminates the need for carbidopa, and, as they are no longer required, removes the signs, symptoms, side effects, or adverse reactions associated with carbidopa or benserazide in virtually all patients. With the removal of carbidopa, the risks and problems associated with peripheral depletion of the centrally acting monoamines are eliminated, which is a great safety advantage.

L-dopa is an amino acid that may be classified by the US Food and Drug Administration (FDA) as a drug, a medical food, or a nutritional supplement, depending upon the application. As a nutritional supplement, L-dopa is classified by the FDA as Generally Recognized As Safe (GRAS), with a side effect profile safe enough to allow for over-the-counter sales. The combination of L-dopa with carbidopa is only 
Table I Previously published side effects and adverse reactions associated with carbidopa

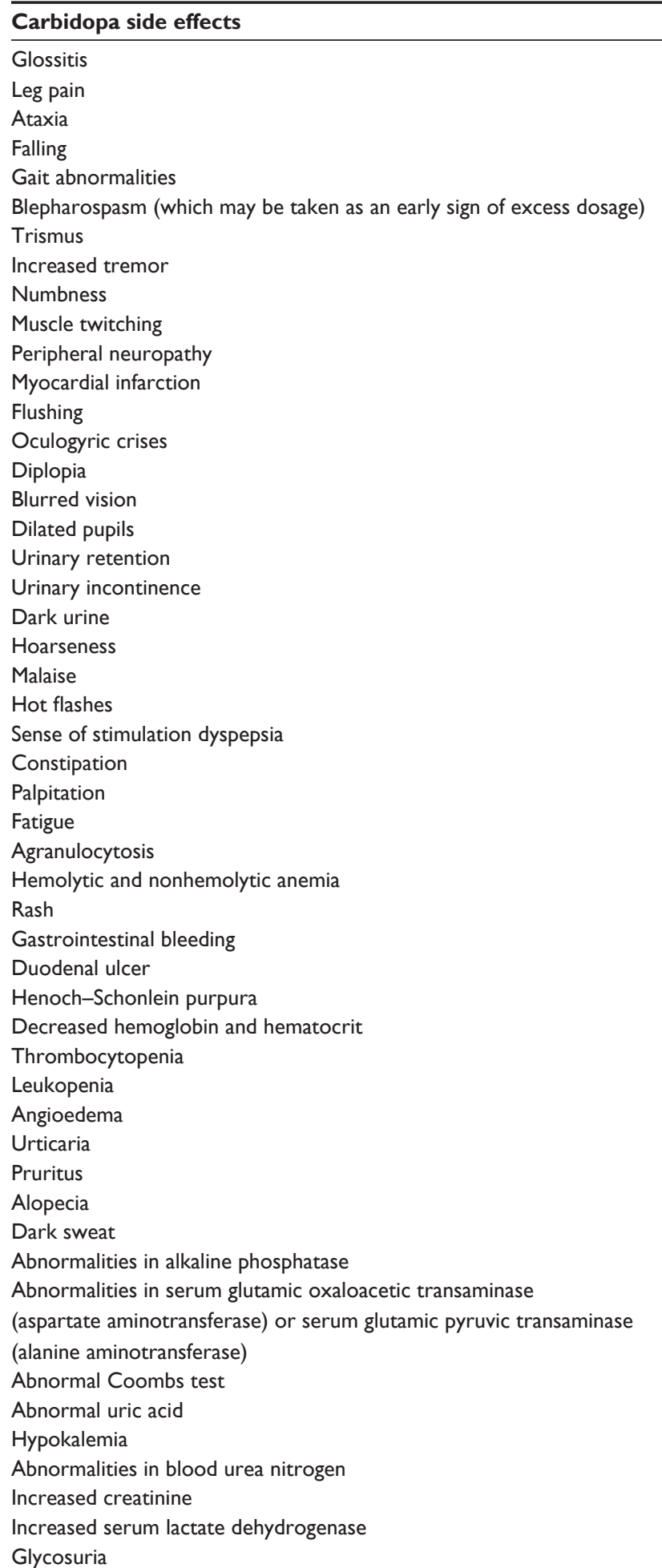

Note: Data from Hinz et al.2.12

classified as a drug; it is not listed as GRAS by the FDA. Currently, in the US, if a patient experiences a carbidopa side effect, the only available form of L-dopa without carbidopa is a nutritional supplement product containing standardized
L-dopa. It is the experience of Hinz et al ${ }^{2,12}$ that few physicians are aware of the availability of the nutritional supplement form of standardized L-dopa over the counter in the US, and even fewer understand the management of L-dopainduced nausea without the use of carbidopa.

Table 1 is a previously published list of side effects and adverse reactions associated with peripheral depletion of centrally acting monoamines (serotonin, dopamine, norepinephrine, and epinephrine) due to carbidopa administration. ${ }^{2,12}$

The current standard of care for Parkinson's disease is based on the endogenous state perspective. There is no consideration that nausea is caused by the imbalance between the serotonin and dopamine systems. The depletions of serotonin, thiols, L-tyrosine, L-tryptophan, and other monoamines associated with the clinical course of Parkinson's disease, L-dopa monotherapy, and the use of general decarboxylase inhibitors are not addressed (see Table 2).,12

Under the current standard of care, the etiology of the signs and symptoms associated with these depletions is not adequately recognized, understood, or controlled. Standard treatment of Parkinson's disease under endogenous conditions is to simply increase L-dopa/carbidopa if symptoms of Parkinson's disease are not optimally under control.

Competitive inhibition research has identified the causes of the depletion and previously published the steps required to increase the synthesis in a properly balanced manner,

Table 2 Depletions of centrally acting monoamines (serotonin, dopamine, norepinephrine, and epinephrine), thiols, L-tyrosine, and L-tryptophan associated with Parkinson's disease, L-dopa administration, and administration of a general decarboxylase inhibitor

\begin{tabular}{|c|c|c|c|}
\hline & $\begin{array}{l}\text { Parkinson's } \\
\text { disease }\end{array}$ & $\begin{array}{l}\text { L-dopa } \\
\text { administration }\end{array}$ & $\begin{array}{l}\text { General } \\
\text { decarboxylase } \\
\text { inhibitor }\end{array}$ \\
\hline Serotonin & $\begin{array}{l}\text { Depletion } \\
\text { known }\end{array}$ & Depletion known & $\begin{array}{l}\text { Peripheral } \\
\text { depletion known }\end{array}$ \\
\hline Dopamine & $\begin{array}{l}\text { Depletion } \\
\text { known }\end{array}$ & & $\begin{array}{l}\text { Peripheral } \\
\text { depletion known }\end{array}$ \\
\hline Norepinephrine & $\begin{array}{l}\text { Depletion } \\
\text { known }\end{array}$ & & $\begin{array}{l}\text { Peripheral } \\
\text { depletion known }\end{array}$ \\
\hline Epinephrine & $\begin{array}{l}\text { Depletion } \\
\text { known }\end{array}$ & & $\begin{array}{l}\text { Peripheral } \\
\text { depletion known }\end{array}$ \\
\hline Thiols & $\begin{array}{l}\text { Depletion } \\
\text { known }\end{array}$ & Depletion known & \\
\hline L-tyrosine & $\begin{array}{l}\text { Depletion } \\
\text { known }\end{array}$ & Depletion known & \\
\hline L-tryptophan & $\begin{array}{l}\text { Depletion } \\
\text { known }\end{array}$ & Depletion known & \\
\hline
\end{tabular}

Note: Adapted with permission from Dove Medical Press. Hinz M, Stein A, Uncini T. Relative nutritional deficiencies associated with centrally acting monoamines. Int J Gen Med. 2012;5:413-430.12 Copyright (c) 2012.

Abbreviation: L-dopa, L-3,4-dihydroxyphenylalanine. 
leading to optimal functional results. The properly balanced competitive inhibition approach avoids the extensive depletion of serotonin, thiols, L-tyrosine, and L-tryptophan that is known to exist with L-dopa monotherapy. It also eliminates the nausea dosing barrier that may occur when L-dopa is administered without the need for a general decarboxylase inhibitor. ${ }^{2,12}$

\section{Materials and methods}

A total of 813 medical patients with a diagnosis of Parkinson's disease were queried from a database owned by DBS Labs (Duluth, MN, USA). These were patients who had collected urine samples in the competitive inhibition state and then submitted them for serotonin and dopamine assay followed by OCT2 functional status determination. , $2,13-20^{2}$

The Parkinson's disease patients' diagnostic evaluations were performed under the care of a licensed medical doctor or doctor of osteopathic medicine and then entered as a working diagnosis on submission of laboratory samples. The diagnosis of Parkinson's disease was then added to the database without further diagnostic verification.

Patient demographics are as follows. Total number of Parkinson's disease patients included for consideration in this paper: $\mathrm{N}=813$ of which males were $\mathrm{N}=554$ (68.14\%) and females were $\mathrm{N}=259$ (31.86\%). The male age range was 42-95 years with a mean of 70 years and a standard deviation of 10.0 years. The female age range was 28-91 years with a mean of 66 years 8 months and a standard deviation of 10.6 years.

Amino acid formulas were obtained from CHK Nutrition (Duluth, MN, USA). The following formulas were utilized:

- NeuroReplete (eight pills containing 5-HTP 99\% + pure $300 \mathrm{mg}$, L-tyrosine 3,000 mg, L-lysine $500 \mathrm{mg}$, vitamin C $1,000 \mathrm{mg}$, vitamin B6 $75 \mathrm{mg}$, calcium carbonate $220 \mathrm{mg}$, and folate $400 \mu \mathrm{g}$ )

- D5 Mucuna $300 \mathrm{mg}$ pills of 40\% L-dopa standardized (each pill containing $120 \mathrm{mg}$ L-dopa)

- D5 Mucuna powder (one level tablespoonful [2.4 g] containing $840 \mathrm{mg}$ L-dopa)

- CysReplete (six pills containing L-cysteine 4,500 mg, selenium $400 \mu \mathrm{g}$, and folate $400 \mu \mathrm{g}$ ).

The patients were started on one pill of NeuroReplete in the morning and at $4 \mathrm{pm}$ to achieve 5-HTP control of L-dopa-induced dopamine and serotonin depletion symptoms, including nausea and/or vomiting. If nausea and/or vomiting become a problem, the 5-HTP daily dosing value is addressed by adjusting the NeuroReplete within the range of 37.5-600 mg per day until the symptoms are controlled. As 5-HTP levels can be either high or low relative to L-dopa for nausea control, the first adjustment is to decrease the 5-HPT intake by $37.5 \mathrm{mg}$ per day. If that change is not effective, at 3-day intervals the 5-HTP level is increased in daily incremental values going up to $112.5 \mathrm{mg} /$ day, then up $150 \mathrm{mg}$ per day, then $300 \mathrm{mg}$ per day, then up to a maximum of $600 \mathrm{mg}$ per day. No patients $(\mathrm{N}=813)$ experienced nausea that was refractory to this 5-HTP approach.

With regard to L-dopa administration, patients were started on two pills of D5 Mucuna 40\% in the morning, noon, and at $4 \mathrm{pm}$. The D5 Mucuna 40\% was then increased weekly in six-pill increments (L-dopa daily dosing value increases of $720 \mathrm{mg}$ ) until symptoms were brought under control or an L-dopa daily dosing value of 6,720 mg was achieved, whichever came first. If there was no symptom relief at $6,720 \mathrm{mg}$ per day, a pill stop, as outlined in the following section, was started in order to identify whether the daily L-dopa dosing value was overdosed or underdosed relative to optimal therapeutic dosing. The optimal therapeutic range for the daily L-dopa dosing was from $720 \mathrm{mg}$ to $16,800 \mathrm{mg}$ per day with a mean of 5,880 $\mathrm{mg}$ per day and a standard deviation of $1,190 \mathrm{mg}$.

All patients were started on two pills of CysReplete three times a day, with the first dose at noon to prevent and/or reverse thiol depletion associated with Parkinson's disease and/or the administration of L-dopa. The daily L-cysteine dose was static and not adjusted. For a discussion of the establishment of the static dosing requirements of the CysReplete formula, the reader is referred to prior writings of Hinz et al (2009). ${ }^{12}$

\section{The pill stop protocol}

If the patient was experiencing residual symptoms associated with Parkinson's disease when the daily dosing value of L-dopa was established at 6,720 mg per day (equal to 56 pills each containing $120 \mathrm{mg}$ of L-dopa), a 2-day pill stop of all amino acids was implemented. This was utilized to define whether the patient's daily L-dopa intake was too high or too low relative to the optimal therapeutic dosing value.

With each pill stop, one of three general outcomes was typically observed:

1. If in the morning following the first day of a complete pill stop the patient's Parkinson's disease symptoms, from the patient's perspective, were markedly improved, it was interpreted that the patient was overdosed relative to the optimal daily dosing value requirements.

2. If in the morning following the first day of a complete pill stop the patient's Parkinson's disease symptoms were the same or worse, it was interpreted that the patient's daily 
L-dopa dosing value was too low relative to the optimal therapeutic requirements.

3. If a patient experienced a deterioration of symptoms the same day that the pill stop was initiated, all amino acids should be restarted immediately at the previous daily dosing values, as the patient was underdosed.

A patient's daily L-dopa dosing value was considered to be optimal when it corresponded with the greatest relief of symptoms. At that point, no further pill stops were required.

For those patients who did not achieve optimal symptom relief after the first pill stop, subsequent pill stops were undertaken. The patient who reported relief of symptoms the morning following the pill stop was designated as being given an L-dopa overdose relative to the optimal dosing needs. The overdosed value was then referenced against the daily L-dopa dosing value of the most recent previous pill stop where the patient underdosed. With these high and low values recorded, the optimal L-dopa dosing was then defined. The patient was placed on the higher daily L-dopa dosing value minus $240 \mathrm{mg}$ per day of L-dopa and evaluated again in 7 days. If symptoms were not at the level experienced the morning after the pill stop when the L-dopa was overdosed, the daily dosing value was decreased another $240 \mathrm{mg}$ per day. This combination of pill stops with decreases of $240 \mathrm{mg}$ L-dopa daily dosing values was continued until optimal relief of Parkinson's disease symptoms was achieved. Symptomatic relief should be on a par with the marked improvement experienced the morning after the initial pill stop where the L-dopa overdose relative to optimal therapeutic needs was identified.

Those patients who failed to show improvement the morning after the pill stop were interpreted as having been administered L-dopa daily dosing values that were too low relative to the required optimal dosing needs. The L-dopa daily dosing value was then increased by $720 \mathrm{mg}$ and another pill stop was performed in 1 week.

The pill stop criteria require answering the following questions from the patient's perspective with regard to overall Parkinson's disease symptoms: whether symptoms were better, whether symptoms were worse, or whether symptoms were the same.

A patient's response to a question is not always direct. When the caregiver is not confident in the response to the questions, it is recommended that another pill stop be performed. One physician reported performing three pill stops with a patient on the same daily L-dopa dosing value before being convinced that the proper clinical data were in place to make a dosing change decision.

\section{Results}

The pill stop concept evolved from initial observations where Parkinson's disease patients taking higher daily dosing values of L-dopa ( $>10,800 \mathrm{mg}$ ) had either missed pills or stopped their pills during treatment. Physicians reported patients who in the morning of the day following the stopping of all amino acid pills experienced what turned out to be a period of optimal symptom relief. A brief period of time (3-6 hours) was noted with a remarkable improvement from the patient's perspective. These patients spontaneously volunteered comments such as "This is the best I have felt in years" or "For 20 years I have wanted to feel this good". The comments were definitive. They clearly indicated that from the patient's perspective an abrupt dramatic and positive change in the patient's symptoms had occurred. It was subsequently determined that in these patients the daily L-dopa dosing value in the competitive inhibition state prior to the L-dopa pill stop was too high. These patients had been unknowingly overdosed. When all amino acids are stopped, systemic L-dopa and dopamine levels decrease through the levels that are required for optimal control of symptoms. A period of optimal symptom relief occurs approximately 24 hours after the pill stop where the first L-dopa dosing was missed.

Most surprising was the novel observation in the competitive inhibition state. Identical Parkinson's disease symptoms of the same intensity were present when L-dopa daily dosing values were too high or too low relative to optimal daily dosing value. Typically, it is clinically impossible to determine whether the patient's daily L-dopa dosing value is too high or too low without a pill stop. An L-dopa overdose cannot be determined based on traditional signs and symptoms observed in the endogenous state. These novel clinical overdose observations do not exist in the endogenous state, and observations in the endogenous state do not have predictability with regard to outcomes of amino acid administration in the competitive inhibition state. When administering properly balanced L-dopa with 5-HTP, L-tyrosine, and thiols in the competitive inhibition state, this novel pill stop approach is required to prevent L-dopa overdose and to assist in identifying the optimal therapeutic dosing range. ${ }^{12}$

As noted in Figure 1, there is an L-dopa daily dosing value range where symptoms are optimally controlled. This dosing range is very narrow: $\pm 240 \mathrm{mg}$ relative to the optimal therapeutic value. The point of optimal symptom relief is indicated with an " $X$ ". Figure 1 also illustrates the 


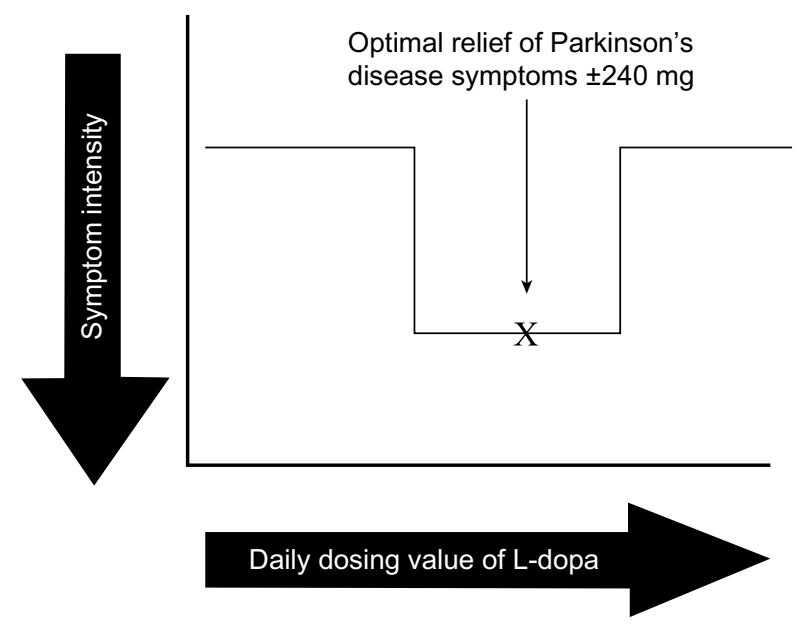

Figure I The typical dose-response curve observed with administration of L-dopa in the competitive inhibition state (concomitant administration of L-dopa, 5-hydroxytryptophan, a thiol, and L-tyrosine).

Notes: There is an abrupt cessation or return of symptoms when the daily dosing value of $\mathrm{L}$-dopa is too high or too low. The dosing value associated with these abrupt changes is small, generally $120 \mathrm{mg}$ per day or less. The range associated with optimal relief of symptoms is narrow: $\pm 240 \mathrm{mg}$ from the mean.

Abbreviation: L-dopa, L-3,4-dihydroxyphenylalanine.

phenomenon observed with this narrow optimal dosing value range where symptoms abruptly resolve or return with small increases or decreases of the daily L-dopa dosing value $(\leq 120 \mathrm{mg}$ ). When these inflection points are reached, it is not a gradual resolution or return of symptoms. The change in symptoms tends to be abrupt.

Changing the daily L-dopa dosing value by $120 \mathrm{mg}$ can have dramatic clinical results. In general, this is independent of the size of the daily L-dopa dose. For example, a patient was taking $10,800 \mathrm{mg}$ of L-dopa per day (equivalent to 90, $120 \mathrm{mg}$ L-dopa pills) in the competitive inhibition state. The patient reported being frozen in the chair and unable to stand. After a pill stop the patient was placed on 89 pills per day (10,680 mg of L-dopa). After a daily decrease in the L-dopa dosing value of only $120 \mathrm{mg}$, the patient was able to rise without assistance and ambulate. These results are common, not rare.

No L-dopa discussion relative to Parkinson's disease would be complete without touching on the topic of dyskinesias. In the competitive inhibition state, no problems or concerns were noted with dyskinesias under this approach in the 10 years of implementation. Further discussion is reserved for other papers.

\section{Discussion}

The novel focus of this paper is that in the competitive inhibition state L-dopa daily dosing values that are too high or too low relative to the optimal therapeutic range manifest the same symptoms with identical intensity. This phenomenon is so pervasive that pill stop evaluation needs to be conducted with all patients if optimal relief of symptoms is not achieved when the daily dosing value is increased to a specific set point. The pill stop should be performed if relief of symptoms has not been achieved at L-dopa daily dosing values $\geq 6,720 \mathrm{mg}$ per day, or if a question exists regarding the direction of the next change in the L-dopa daily dosing value. It is impossible to empirically determine with absolute certainty whether patients in the competitive inhibition state are taking too much or too little L-dopa without a pill stop. The only exception is if the L-dopa daily dosing value happens to be established at the optimal therapeutic value during a dosing adjustment. Blindly increasing the daily L-dopa dosing values in a linear manner based on endogenous reference points (status of symptoms) in the competitive inhibition state has a high potential for L-dopa overdose relative to the optimal therapeutic dosing value.

In the competitive inhibition state, the daily L-dopa dosing value range where optimal relief of symptoms is obtained is as narrow as $\pm 120 \mathrm{mg}$ of L-dopa in some patients. With L-dopa daily dosing value increases of $720 \mathrm{mg}$ or more, it is common to exceed the optimum dosing value, leading to an overdose situation.

\section{Conclusion}

This paper is about safety, not efficacy, of L-dopa. Efficacy has been established by numerous studies over the last 50 years it has been administered. The enhanced safety margin is related to L-dopa overdose management.

This paper reports a novel observation relating to L-dopa in the competitive inhibition state. L-dopa daily dosing values that are either excessive or insufficient relative to the optimal therapeutic requirements are clinically associated with the exact same symptoms of Parkinson's disease, each with identical intensity. These novel findings document that there are no clinical signs or symptoms for the physician to formulate a conclusion that the patient is overdosed on L-dopa and is above the optimal therapeutic dosing range.

From a safety standpoint, the pill stop is required in the competitive inhibition state to prevent L-dopa overdose and facilitate realization of the therapeutic dosing value. It has been previously documented how depletions of serotonin, L-tyrosine, and thiols are associated with Parkinson's disease and potentiated by L-dopa monotherapy with or without a general decarboxylase inhibitor in the endogenous state. Peripheral depletion of serotonin, dopamine, norepinephrine, and epinephrine is facilitated by administration of carbidopa 
or benserazide. If these depletion issues are to be addressed properly, the patient has to be placed in the competitive inhibition state, and L-dopa daily dosing value needs to be guided by pill stops.

The purpose of this paper is to outline a novel safety concern identified with administration of L-dopa in the competitive inhibition state that has not been previously described in the literature and to facilitate discussion of these findings.

\section{Disclosure}

Marty Hinz discloses his relationship with DBS Labs, Inc. and NeuroResearch Clinics, Inc. The other authors report no conflicts of interest in this work.

\section{References}

1. Hinz M, Stein A, Uncini T. Validity of urinary monoamine assay sales under the "spot baseline urinary neurotransmitter testing marketing model". Int J Nephrol Renovasc Dis. 2011;4:101-113.

2. Hinz M, Stein A, Uncini T. Amino acid management of Parkinson disease: a case study. Int J Gen Med. 2011;4:1-10.

3. Vieira-Coelho M, Soares-Da-Silva P. Apical and basal uptake of L-dopa and 5-HTP and their corresponding amines dopamine and 5-HT in OK cells. Am J Physiol. 1997;272(5 Pt 2):F632-F639.

4. Wang Z, Srragy H, Felder R, Carey R. Intrarenal dopamine production and distribution in the rat: physiological control of sodium excretion. Hypertension. 1997;29:228-234.

5. Suzuki H, Nakane H, Kawamura M, Yoshizawa M, Takeshita E, Saruta T. Excretion and metabolism of dopa and dopamine by isolated perfused rat kidney. The American Physiological Society. 1984:E285-E290.

6. Adam W, Drangova R. Production and excretion of dopamine by the isolated perfused rat kidney. Renal Physiol. 1985;8:150-158.

7. Kambara S, Yoneda S, Yoshimura M, et al. The source and significance of increased urinary dopamine excretion during sodium loading in rats. Nippon Naibunpi Gakkai Zasshi. 1987;63(5):657-663.

8. Zimlichman R, Levinson P, Kelly G, Stull R, Keiser H, Goldstein D. Derivation of urinary dopamine from plasma dopa. Clin Sci (Lond). 1988;75(5):515-520.
9. Carey R. Renal dopamine system: paracrine regulator of sodium homeostasis and blood pressure. Hypertension. 2001;38:297-302.

10. Hagege J, Richet G. Proximal tubule dopamine histofluorescence in renal slices incubated with L-dopa. Kidney Int. 1985;27(1):3-8.

11. Isaac J, Berndt TJ, Knox FG. Role of dopamine in the exaggerated phosphaturic response to parathyroid hormone in the remnant kidney. J Lab Clin Med. 1995;126:470-473.

12. Hinz M, Stein A, Uncini T. Relative nutritional deficiencies associated with centrally acting monoamines. Int J Gen Med. 2012;5:413-430.

13. Hinz M, Stein A, Uncini T. APRESS: apical regulatory super system, serotonin, and dopamine interaction. Neuropsychiatr Dis Treat. 2011;7:1-7.

14. Hinz M, Stein A, Uncini T. The dual-gate lumen model of renal monoamine transport. Neuropsychiatr Dis Treat. 2010;6:387-392.

15. Stein A, Hinz M, Uncini T. Amino acid responsive Crohn's disease: a case study. Clin Exp Gastroenterol. 2010;3:171-177.

16. Hinz M, Stein A, Uncini T. Treatment of attention deficit hyperactivity disorder with monoamine amino acid precursors and organic cation transporter assay interpretation. Neuropsychiatr Dis Treat. 2011;7:31-38.

17. Hinz M, Stein A, Uncini T. Urinary neurotransmitter testing: considerations of spot baseline norepinephrine and epinephrine. Open Access J Urol. 2011;3:19-24.

18. Hinz M, Stein A, Uncini T. Monoamine depletion by reuptake inhibitors. Drug Healthc Patient Saf. 2011;3:69-77.

19. Hinz M, Stein A, Uncini T. The discrediting of the monoamine hypothesis. Int J Gen Med. 2012;5:135-142.

20. Hinz M, Stein A, Uncini T. 5-HTP efficacy and contraindications. Int J Gen Med. 2012;5:413-430.

21. Barbeau A. The pathogenesis of Parkinson's disease: a new hypothesis. Can Med Assoc J. 1962;87(15):802-807.

22. Mayo Clinic. Parkinson's disease. Treatment and drugs. Available from: http://www.mayoclinic.org/diseases-conditions/parkinsons-disease/ basics/treatment/con-20028488. Accessed June 19, 2014.

23. Critchley E. L-dopa and carbidopa (sinemet) in the management of parkinsonism. Postgrad Med J. 1975;51:619-621.

24. Sinemet $\mathrm{CR}^{\circledR}$ (carbidopa-levodopa) [prescribing information]. Merck Sharp \& Dohm Corp. Available from http://dailymed.nlm.nih.gov/ dailymed/lookup.cfm?setid=69e575b9-f8a5-494f-b736-2520ef505cb0. Accessed July 1, 2014.
Drug, Healthcare and Patient Safety

\section{Publish your work in this journal}

Drug, Healthcare and Patient Safety is an international, peer-reviewed open-access journal exploring patient safety issues in the healthcare continuum from diagnostic and screening interventions through to treatment, drug therapy and surgery. The journal is characterized by the rapid reporting of reviews, original research, clinical, epidemiological and

\section{Dovepress}

post-marketing surveillance studies, risk management, health literacy and educational programs across all areas of healthcare delivery. The manuscript management system is completely online and includes a very quick and fair peer-review system. Visit http://www.dovepress.com/ testimonials.php to read real quotes from published authors. 